\title{
Mathematical problems related to the location of the measurement and the date of measurement
}

\author{
Yang Liu \\ North China Electric Power University, Huadian-Lu 689, 071000 Baoding,China \\ 2228190224@163.com
}

Keywords:shadow, The optimal direction estimation,location.

\begin{abstract}
We could take this subject as a mathematical project which is based on known measurement time and shadow vertex coordinate data, then solve the measurement location and date questions. We shall transform it into an optimal solution of the optimization problem, the constraint condition and objective function are same as sin subject 2, decision variable are longitude, latitude, date and height of the pole.
\end{abstract}

\section{Introduction}

Take the value of a set of longitude, latitude and date as the initial point. In three dimensional space(The $\mathrm{X}$ axis represents the longitude, the $\mathrm{Y}$ axis represents the latitude, and the $\mathrm{Z}$ axis represents the date), search in 26 directions with a certain step length. Then find an optimal direction to get the next basic point. Assuming a straight rod height between $1 \mathrm{~m}$ to $5 \mathrm{~m}$, each height value of a straight bar can be obtained an optimal point and a value of the objective function. As for data of affix 2, we find out when the bar is 1 meters high, the objective function value is minimum, the most optimal point of the correspondence shall be: 89 degrees east longitude, and 36 degrees of south latitude. January 15 th is the place and date of the request. According to data of attachment 3 , we find out when the bar is 2.9 meters high, the objective function value is minimum, the most optimal point of the correspondence shall be: 121.7 degrees east longitude, and 50 degrees of south latitude. May 16th is the place and date of the request ${ }^{[1]}$.

\section{Model assumptions}

a) Assuming that the sun's rays on the earth's surface are parallel to the light.

b) Assuming that the straight bar is located in the ground plane 。

c) Assuming that the length of the straight rod is not affected by the external factors such as temperature, pressure and so on.

d) Assuming that the earth is a ball, and place on earth level ground is the section of surface sphere to the plane.

e) Without taking into account the refraction of the sun's rays through the atmosphere, the surface of the sun, the high mountain barrier, and the elevation and other factors. 


\section{Symbol Description}

\begin{tabular}{|c|c|c|c|}
\hline Symbol & Significance & Symbol & significance \\
\hline$a$ & elevation angle of the sun & $\psi$ & longitude \\
\hline$\square \alpha$ & solar aspect angle & $h$ & Time of Beijing \\
\hline$\square \delta$ & declination & $E$ & time difference \\
\hline$\square \omega$ & angel of equator and latitude & $L$ & straight rod length \\
\hline$\varphi$ & solar hour angle & l & Length of straight bar \\
\hline$\square\left(\mathrm{x}_{\mathrm{i}}, \mathrm{y}_{\mathrm{i}}\right)$ & latitude & $\left(\mathrm{X}_{\text {yesi }}, \mathrm{Y}_{\text {yesi }}\right)$ & Length of the shadow of straight bar \\
\hline
\end{tabular}

\section{Establishment and Solution Model}

Subject three can be taken as a mathematical project which is based on known measurement time and shadow vertex coordinate data measured at different times, then solve the measurement location and date questions. We could transform it into an optimal solution of the optimization problem, establish nonlinear programming model that takes longitude, latitude, and date as decision variable, and the position and the actual position of the shadow of the straight bar as the objective function[2].

\subsection{Establishment of model}

The constraint conditions and objective functions of the model are the same as the 6.1 model. Decision variables are: latitude, latitude, and accretion, and a nonlinear programming model is obtained:

$$
\begin{gathered}
\min f=\sum_{i=1}^{N}\left[\left(x_{i}-x_{\text {yesi }}\right)^{2}+\left(y_{i}-y_{\text {yesi }}\right)^{2}\right] \\
w_{i}=(h+E) \times 15+\psi-300 \\
E=\frac{\psi-120}{15} \\
\sin a_{i}=\sin \varphi \sin \delta+\cos \varphi \cos \delta \cos \omega_{i} \\
\sin \alpha_{i}=\frac{\cos \delta \sin \omega_{i}}{\cos a_{i}} \\
L \\
l_{i}=\frac{\tan \left[\arcsin \left(\sin \varphi \sin \delta+\cos \varphi \cos \delta \cos \omega_{i}\right)\right]}{x_{i}=l_{i} \cos \alpha_{i}} \\
y_{i}=l_{i} \sin \alpha_{i} \\
1 \leq i \leq N
\end{gathered}
$$

\section{2 solution of the model}

For the extremum problem of single objective constrained that mentioned above, we shall take reference of the solution of subject 2. First, it is transformed into a single objective unconstrained extremum problem, and then use the improved search algorithm to solve the problem, the algorithm is described as follows[3].

Step 1: take an optional initial approximate point $B_{1}$, then explore with the initial basis point (the first basis point) 
Step 2: choose a step length $\lambda_{i}(i=1,2,3,4 \ldots \ldots)$ for each direction we search $p_{i}(i=0,1,2,3 \ldots \ldots)$

Step 3: count $f\left(B_{j}+\lambda_{i}\right), i=1,2,3 \ldots \ldots 26$, from the very first basic point J, (J start from 1), if there is a $i=k$, and it makes $f\left(B_{j}+\lambda_{i}\right)$ as minimum in No26, and $f\left(B_{j}+\lambda_{i}\right)<f\left(B_{i}\right)$, then the basic

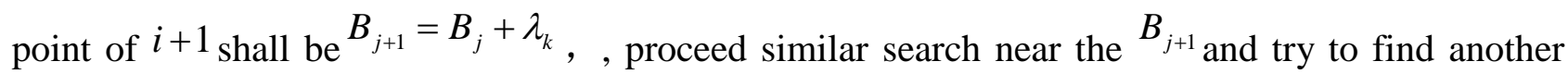
basic point, if there is no $\mathrm{i}$, the record the value of $B_{j}$, and move forward to step 4.

Step 4: find the optimal objective function value $f\left(B_{j}\right)$ by ${ }^{B_{j}}$; Take a certain length of step, then get a set of located in the interval value $\left[L_{\min }, L_{\max }\right]$, find optimal objective function value of each $\mathrm{L}$, then compare these values and the Land ${ }^{B_{j}}$ shall be the final optimal value of minimum value[4].

For the shadow vertex coordinates data, shall to write the corresponding algorithm program by taking help of MATLAB. The interval of height of straight bar is taken as [1,5], start the search by the initial point of 120 degrees in longitude, 20 degrees in north latitude and the date of 19th July, then we can get the height of straight bar is $1 \mathrm{~m}$, the location should be 89 degrees east longitude, south latitude 36 degrees, and days for 46, which is 15th January, the search path in the program as shown in figure $1[5]$ :

For the shadow vertex coordinates data, shall to write the corresponding algorithm program by taking help of MATLAB. The interval of height of straight bar is taken as [1,5], start the search by the initial point of 120 degrees in longitude, 20 degrees in north latitude and the date of 19th July, then we can get the height of straight bar is $2.9 \mathrm{~m}$, the location should be 121.7 degrees east longitude, south latitude 50 degrees, and days for 136, which is 16th May, the search path in the program as shown in figure 2 :

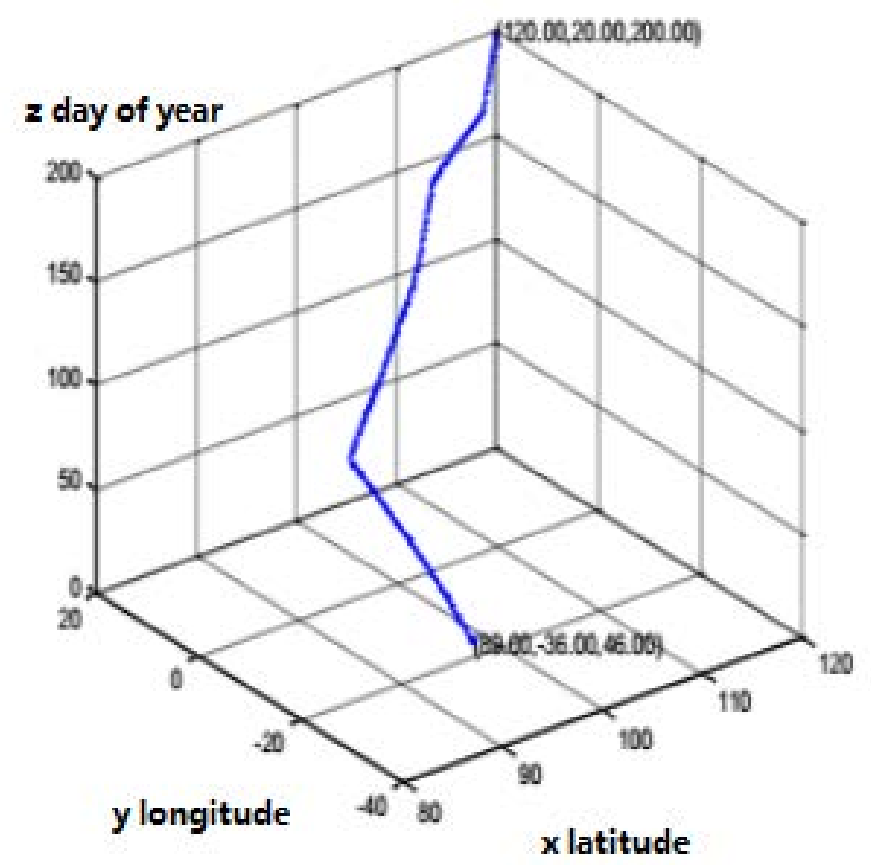

Fig.1 Change of shadow length coordinate in different time(a) 


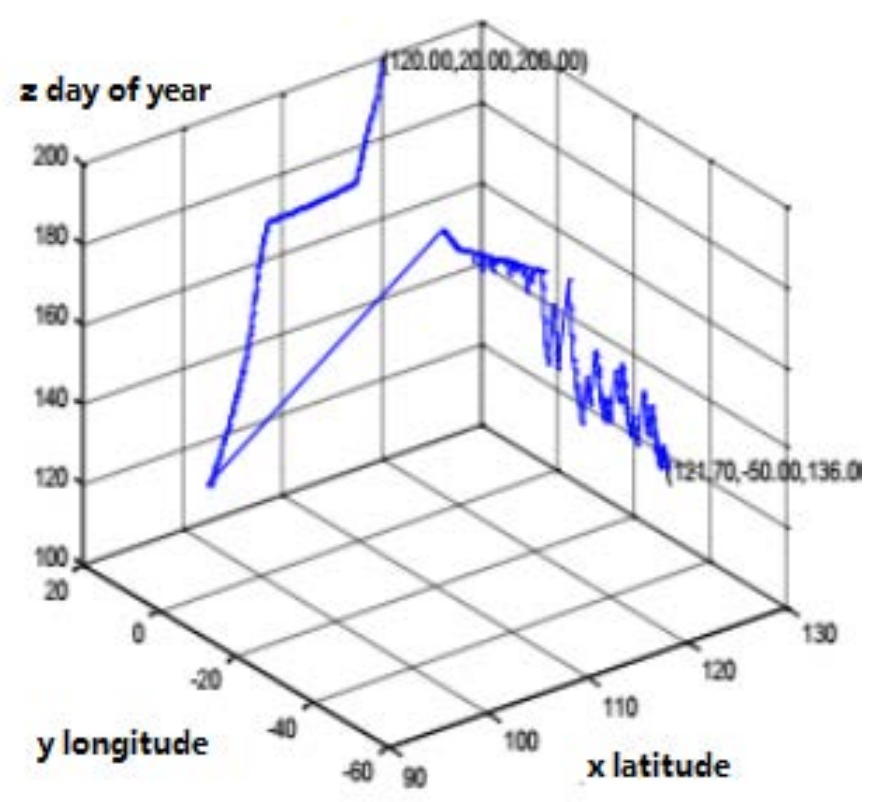

Fig.2 Change of shadow length coordinate in different time(b)

\section{Summary}

In solving the nonlinear programming problem, the search algorithm based on the optimal direction, in a certain extent, improve the convergence speed, but in the solving process is easy to fall into local optimal solution, the solution is not accurate enough.

The search scope of the bar is small, while the calculation of the algorithm is reduced, but it is not accurate enough.

\section{Reference}

[1].Information on http://wenku.baidu.com.

[2].Genshi Lin, using the calculation of the coordinates of the sun to carry out high measurement and positioning, Journal of Nanjing Forestry University, 15 (3), 1991

[3]. Shoukui Si, Xijing Sun, mathematical modeling algorithm and application, National Defense Industry Press, 2011

[4] Jingyun Jia. An analytical method for the determination of astronomical azimuth [J]. China Nuclear Science and technology report. 1996 (00)

[5] Hongbin Ma. A simple and convenient method to measure the angular formula of astronomical azimuth [J]. Journal of Shenyang Gold Institute. 1995 (03) 